\title{
Consecutive Changes in Nerve Conduction Studies after Surgery for Carpal Tunnel Syndrome - Useful Parameters for Evaluating Postoperative Recovery
}

\section{Mikio Muraoka ${ }^{1 *}$ and Kenji Watanabe ${ }^{2}$}

${ }^{1}$ Department of Rehabilitation Medicine, Kameda-Daiichi Hospital, Japan

${ }^{2}$ Department of Orthopedic Surgery, Kameda-Daiichi Hospital, Japan

*Corresponding Author: Mikio Muraoka, Department of Rehabilitation Medicine, Kameda-Daiichi Hospital, Niigata, Japan.
Received: November 11, 2020

Published: December 14, 2020

(C) All rights are reserved by Mikio Muraoka and Kenji Watanabe.

\section{Abstract}

Objective: We quantitatively evaluated indicators of postoperative recovery from surgery for carpal tunnel syndrome (CTS) and determined the most sensitive nerve conduction study [NCS] parameters.

Materials and Methods: NCSs were performed in 50 hands with CTS preoperatively, and at 1 and 3 months postoperatively. Four NCS parameters were assessed: abductor pollicis brevis-distal motor latency (APB-DML), index-distal sensory latency (DSL), 2nd lumbrical - interossei latency difference (2L-INT), and ring finger test (Ring).

Results: The 33 hands in which APB compound muscle action potentials (CMAPs) were preoperatively detected showed significant improvement in APB-DML over time. The 46 hands for which 2L CMAPs were preoperatively detected showed significant improvement in 2L-INT over time, clearly reflecting postoperative recovery. Index-sensory nerve action potentials (SNAPs) and Ring-SNAPs were undetected in 41 hands and 50 hands, respectively, and remained undetected at 3 months in 27 hands and 45 hands, respectively.

Conclusion: Our findings suggest that 2L-INT and APB-DML, in this order, are suitable parameters for evaluating postoperative recovery of CTS, while Index-DSL and Ring using SNAPs are not useful.

Keywords: Carpal Tunnel Syndrome; Nerve Conduction Study; Surgery; Median Nerve; Lumbrical

\section{Abbreviations}

2L-INT: $2^{\text {nd }}$ Lumbrical - Interossei Latency Difference; APB-DML: Abductor Pollicis Brevis-Distal Motor Latency; CMAP: Compound Muscle Action Potential; CTS: Carpal Tunnel Syndrome; DSL: Index-distal Sensory Latency; NCS: Nerve Conduction Study; Ring: Ring Finger Test; SNAP: Sensory Nerve Action Potential.

\section{Introduction}

Carpal tunnel syndrome surgery is mainly performed in patients who are severely affected. Patients who do not experience marked recovery immediately following surgery may question the success of the surgery. Therefore, a quantitative method for assessing postoperative recovery after CTS surgery is needed. In the present study, we determined the most sensitive NCS parameters for evaluating postoperative recovery following CTS surgery.

\section{Materials and Methods}

In 78 patients, 90 open carpal tunnel release surgeries were performed over a 45-month period, and 50 hands of 46 patients were monitored for 3 months. Patients who exhibited marked recovery do not require a 3-month follow-up, and thus these 50 hands belonged to patients with rather severe CTS. Of the 46 patients, 10 were men and 36 were women with a mean age of $74.4 \pm 1.9$ years (range: 51 94). We evaluated 4 NCS parameters: abductor pollicis brevis-distal motor latency (APB-DML), index-distal sensory latency (Index-DSL), $2^{\text {nd }}$ lumbrical - interossei latency difference $(2 \mathrm{~L}$ INT), and ring finger test (Ring), as shown in figure 1 [1-3]. 

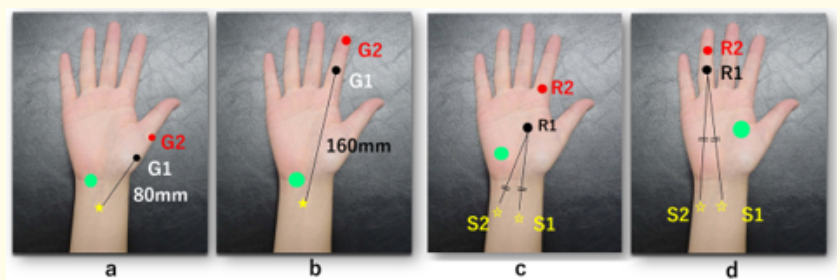

Figure 1: a) APB-DML, b) Index-DSL, c) 2L-INT, d) Ring

\section{Results and Discussion}

Of the 50 hands, 8 were classified with moderate CTS, 25 with severe CTS, and 17 with extreme CTS following Padua's classification, as shown in figure 2 [4]. APB-compound muscle action potentials (CMAPs) were preoperatively undetected in 17 hands; the 33 hands in which APB-CMAPS were detected exhibited significant improvement in APB-DML over time (Figure 3). 2L-CMAPs were preoperatively undetected in only 4 hands and detected in 46 hands. The 46 hands in which 2L-CMAPs were detected showed significant improvement in 2L-INT over time, clearly reflecting postoperative recovery (Figure 4). INT-CMAPs were detected in all 50 hands. Index-sensory nerve action potentials (SNAPs) and Digit 4-SNAPs evoked by median nerve stimulation were undetected in 41 hands and 50 hands, respectively, and remained undetected at 3 months in 27 hands and 45 hands, respectively (Figure 5). Digit 4-SNAPs evoked by ulnar nerve stimulation were detected in all 50 hands.

\section{Nerve Conduction Study}

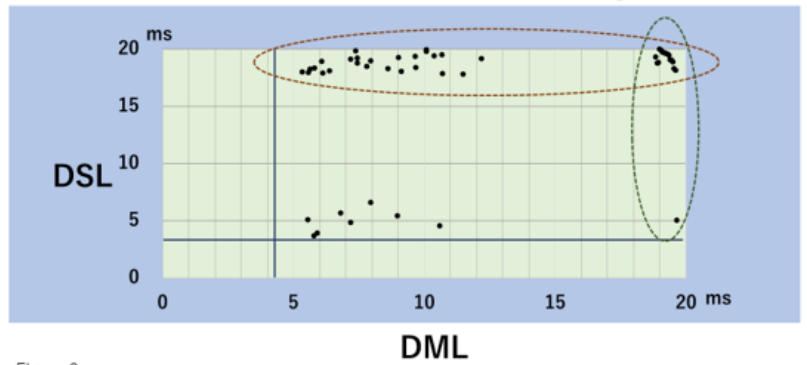

Figure 2: Fifty hands were classified into moderate, severe, or extreme CTS following Padua's classification (cut-off values; $\mathrm{DML}>4.2 \mathrm{~ms}$, DSL $>3.5 \mathrm{~ms}$ ). The dotted circles indicate that parameters were not detected.

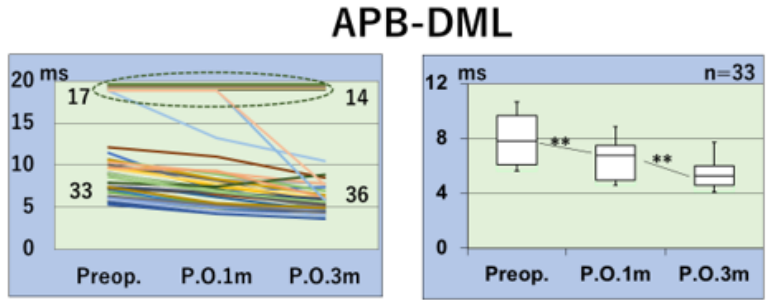

Figure 3: APB-CMAPs were preoperatively undetected in 17 hands, while detected 33 hands consecutively improved APBDML and clearly reflected the postoperative recovery $(\mathrm{P}<0.01$; Wilcoxon t-test with Bonferroni correction).

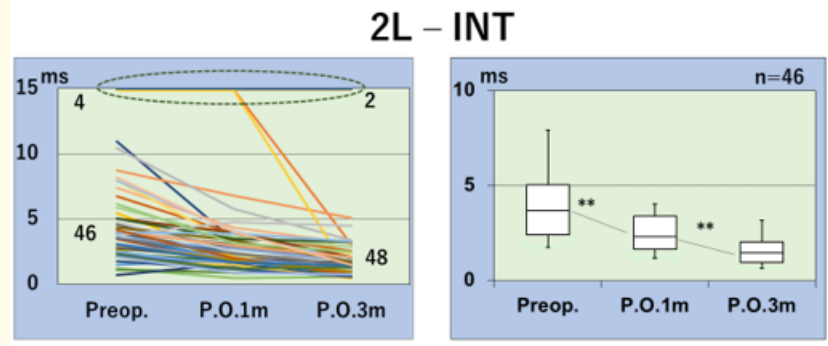

Figure 4: 2L-CMAPs were preoperatively undetected in only 4 hands, and detected in 46 hands, in which 2L-INT showed significant improvement over time, clearly reflecting postoperative recovery $(\mathrm{P}<0.01$; Wilcoxon t-test with Bonferroni correction).

Index-DSL / Digit 4-DSL
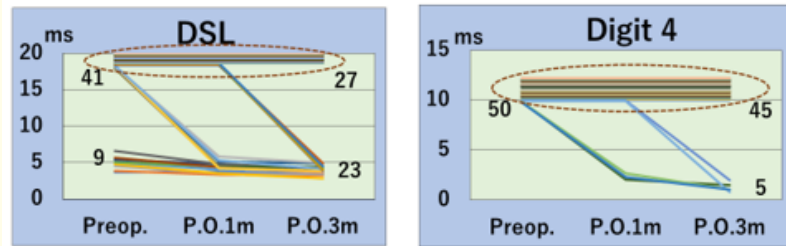

Figure 5: Index-SNAPs and Digit 4-SNAPs [median nerve] were preoperatively undetected in 41 and 50 hands, respectively, and remained undetected in 27 and 45 hands at 3 months, respectively. 
Consecutive Changes in Nerve Conduction Studies after Surgery for Carpal Tunnel Syndrome - Useful Parameters for Evaluating Postoperative Recovery

SNAPs have a short duration, and are susceptible to temporal dispersion in peripheral neuropathy, which is why they are undetectable in severe CTS. Long-lasting CMAPs, on the other hand, are not susceptible to temporal dispersion and are maintained even in severe CTS. For this reason, APB-DML and 2L-INT are useful for determining improvement after CTS surgery [5]. Because the nerve fibers to the 2nd lumbrical are located deep in the carpal tunnel, as shown in the topography of the median nerve (Figure 6), and are thus spared in severe entrapment, 2L-CMAPs are still detected even in extreme CTS [6-10]. Comparison studies including 2L-INT and Ring have high sensitivity because the differences between the latencies detected by median-ulnar stimulation extract the delay at entrapment, which leads to low cut-off values [2,3]. These comparison studies have another advantage in that they exclude errors associated with polyneuropathy [11]. Comparison studies are valuable for evaluating CTS, but in the Ring finger test, SNAPs by radial half digital nerve stimulation show low amplitudes and are often undetected in severe CTS, whereas 2L-INT is suitable for evaluating mild to severe CTS. 2L-INT is strongly recommended and is generally considered the most valuable parameter for predicting postoperative improvement.

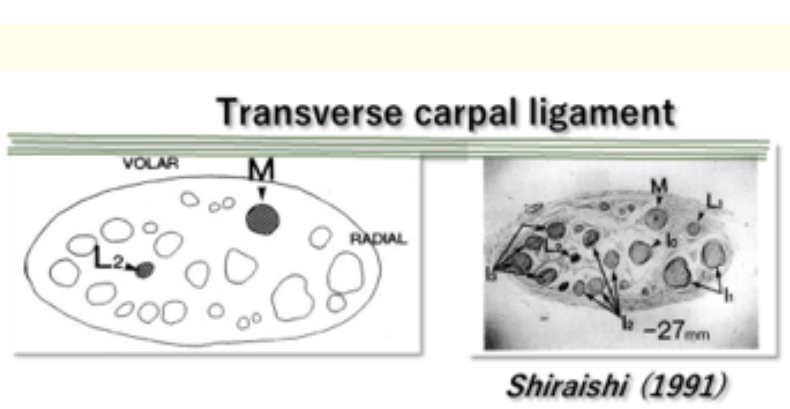

Figure 6: Topography; L2, nerve fibers to 2L; M, nerve fibers to APB.

\section{Conclusion}

Our findings demonstrated that2L-INT and APB-DML, in this order, are suitable parameters for evaluating postoperative recovery of CTS, while Index-DSL and Ring using SNAPs are not useful.

\section{Acknowledgments}

The authors thank Michiko Sakai and Akane Uchiyama for their technical assistance.

\section{Conflict of Interest}

All authors declare no conflicts of interest.

\section{Bibliography}

1. Kohara N. "Clinical and electrophysiological findings in carpal tunnel syndrome”. Brain and Nerve 59 (2007): 1229-1238.

2. Preston D and Logigian E. "Lumbrical and interossei recording in carpal tunnel syndrome". Muscle and Nerve 15 (1992): 1253-1257.

3. Uncini A., et al. "Ring finger testing in carpal tunnel syndrome: a comparative study of diagnostic utility". Muscle and Nerve 12 (1989): 735-741.

4. Padua L., et al. "Neurophysiological classification and sensitivity in 500 carpal tunnel syndrome hands". Acta Neurologica Scandinavica 96 (1997): 211-217.

5. Rotman M., et al. "Time course and predictors of median nerve conduction after carpal tunnel release". Journal of Hand Surgery (American volume) 29 (2004): 367-372.

6. Shiraishi M., et al. "Electrophysiological and anatomical study of the second lumbrical muscle in carpal tunnel syndrome". Journal of Japanese Society for Surgery of the Hand 8 (1991): 269-272.

7. Nobuta S., et al. "Clinical results in severe carpal tunnel syndrome and motor nerve conduction studies". Journal of Orthopaedic Science 10 (2005): 22-26.

8. Logigian E., et al. "Lumbrical sparing in carpal tunnel syndrome: Anatomic, physiologic, and diagnostic implications". Neurology 37 (1987): 1499-1505.

9. Yilmaz F., et al. "Lumbrical-interosseous recording technique versus routine electrodiagnostic methods in the diagnosis of carpal tunnel syndrome". Turkish Journal of Physical Medicine and Rehabilitation 17 (2017): 230-238.

10. Lee S., et al. "Diagnostic value of the second lumbrical-interosseous distal motor latency comparison test in severe carpal tunnel syndrome". Annals of Rehabilitation Medicine 40 (2016): 50-55.

11. Meena A., et al. "Second lumbrical and interossei latency difference in carpal tunnel syndrome". Clinical Neurophysiology 119 (2008): 2789-2794. 


\section{Assets from publication with us}

- Prompt Acknowledgement after receiving the article

- Thorough Double blinded peer review

- Rapid Publication

- Issue of Publication Certificate

- High visibility of your Published work

Website: www.actascientific.com/

Submit Article: www.actascientific.com/submission.php

Email us: editor@actascientific.com

Contact us: +919182824667 